\title{
Favorable Evolution of A Patient With Thromboembolic Pulmonary Hypertension
}

${ }^{1}$ University "Ovidius" of Constanta, Faculty of Medicine

\begin{abstract}
We present a case of severe thromboembolic pulmonary hypertension in a patient with history of recurrent deep vein thrombosis and pulmonary restrictive disease due to pulmonary and vertebral tuberculosis in young adulthood. He was considered not eligible in the National Program for Primary Pulmonary Hypertension, being referred for thoracic surgery, but he was considered unfit for thrombendarterectomy. Despite guidelines, we administered him specific medical therapy (phosphodiesterase-5 inhibitors and endothelin receptor antagonists). His clinical evolution was satisfactory, with increasing effort tolerance and decreasing need for ambulatory oxigenotherapy.
\end{abstract}

Keywords. Pulmonary thromboembolic hypertension, thrombendarterectomy, phosphodiesterase-5 inhibitors, endothelin receptor antagonists.

\section{Irinel Raluca Parepa}

University "Ovidius" of Constanţa, Faculty of Medicine, Department of Cardiology

Aleea Universității 1,900470, Constanța

email : irinel_parepa@yahoo.com

\section{Case Report}

We present the case of a 64 years old male patient, with history of right femuro-popliteal deep vein thrombosis and recurrent pulmonary thromboembolism. He experienced his first pulmonary embolism 3 years ago and repeated a new episode after about 6 months, when we estimated at echocardiography a value of the systolic pressure in the pulmonary artery of $70 \mathrm{mmHg}$, indicating severe pulmonary hypertension.

Due to the recurrence of the pulmonary thromboembolism, at that time we performed investigations aiming to highlight some of the associated thromboembolic risk factors: the thrombophilic profile was normal, tumoral markers, abdominal and pelvic $\mathrm{CT}$ were negative for malignant diseases. He had no family antecedents of clinical manifestations of thromboembolic diseases.

After a period of about 6 months with minimal symptoms under oral anticoagulant and diuretic treatment, the patient returns in the Cardiology Clinic with decompensated right heart failure (inferior limbs edema, hepatomegaly with turgid jugulars), respiratory insufficiency ( spontaneous Sp02 $=88 \%$ ) and systemic hypotension (blood pressure $=90 / 60 \mathrm{mmHg}$, equal on both arms). Clinical examination also revealed severe decreased body mass index $(16,6 \mathrm{~kg} / \mathrm{m} 2)$ and diastolic murmur in the pulmonary area. On ultrasound we detected worsened pulmonary pressures (systolic 
pressure in the pulmonary artery $=120 \mathrm{mmHg}$ ) and marked expansion of right cavities with systolic dysfunction of the right ventricle.

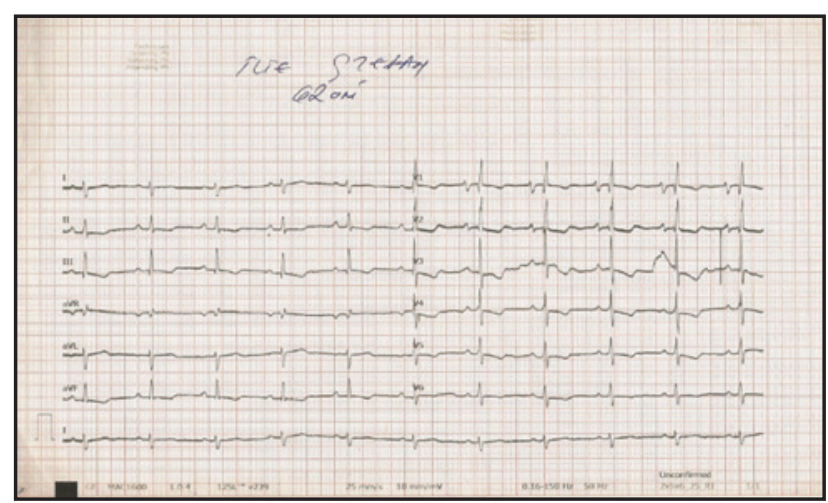

Figure 1. Patient's ECG showing signs of right heart strain

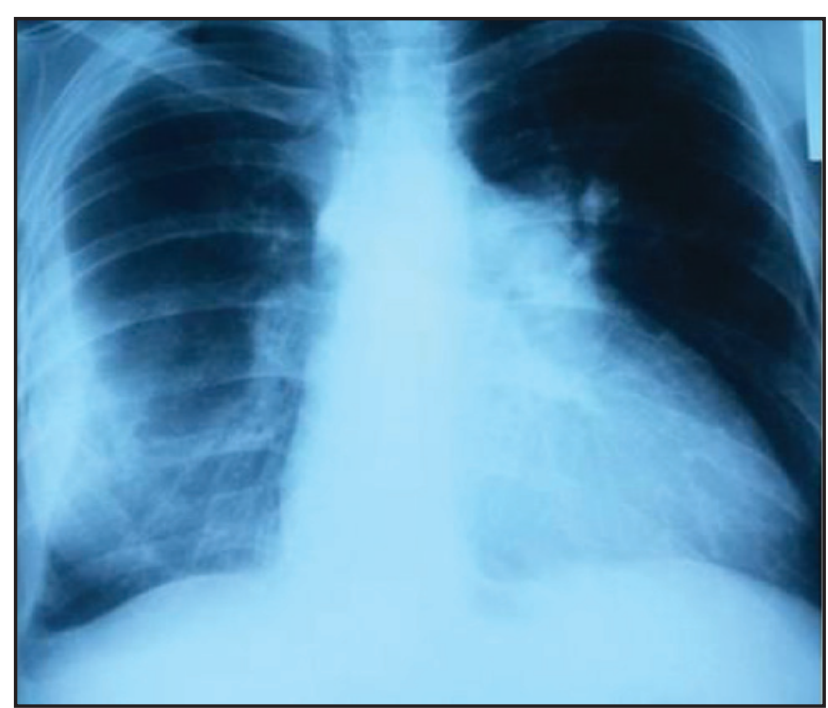

Figure 2. Patient's cardio-pulmonary $x$-ray showing cardiomegaly, mainly on account of the right cavities, right ventricular hypertrophy with dilatation of the pulmonary artery trunk, and left pachypleuritis.

Biological data showed efficientanticoagulation with INR 2,7 and atrial natriuretic peptide highly increased.

The trans-thoracic echocardiography showed a severe dilatation of right cavities, right atrium diameter $72 \mathrm{~mm}$, telediastolic area of the right atrium $37 \mathrm{cmp}$, right ventricle with telediastolic diameter
$58 \mathrm{~mm}$, hypertrophy of the free wall of the right ventricle $=14 \mathrm{~mm}$, tricuspid severe regurgitation, severe systolic dysfunction of the right ventricle with the systolic excursion of the tricuspid ring plan $=13 \mathrm{~mm}$, gradient between right ventricle and right atrium $=92 \mathrm{mmHg}$, inferior vena cava dilated with inspiratory collapse $<50 \%$ expansion of pulmonary trunk $40 \mathrm{~mm}$ and the possible image of thrombus in right pulmonary branch of the pulmonary artery; the left ventricle with reduced dimensions as compared to the right.

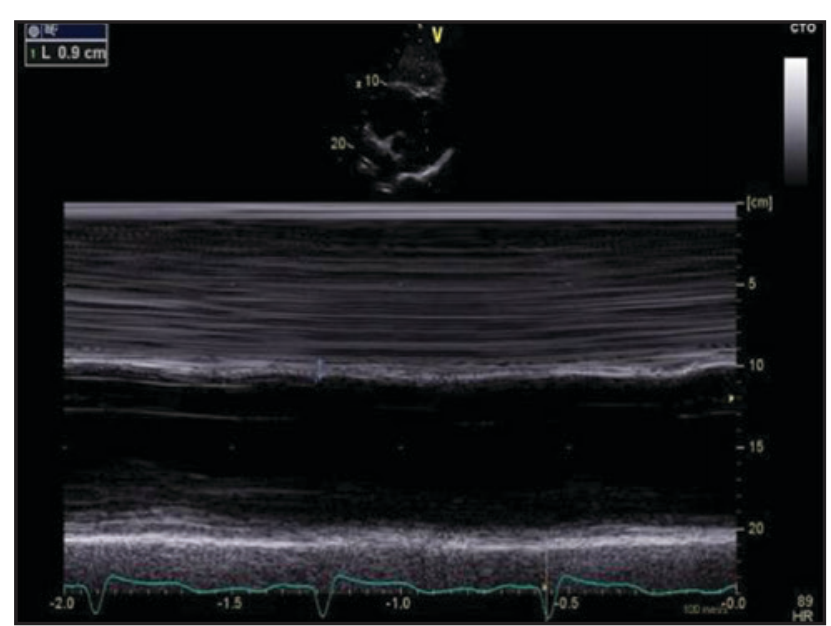

Figure 3. Trans-thoracic echo-cardiography, sub-costal section, M-mode examination underlining right ventricle free wall hypertrophy $(14 \mathrm{~mm})$.

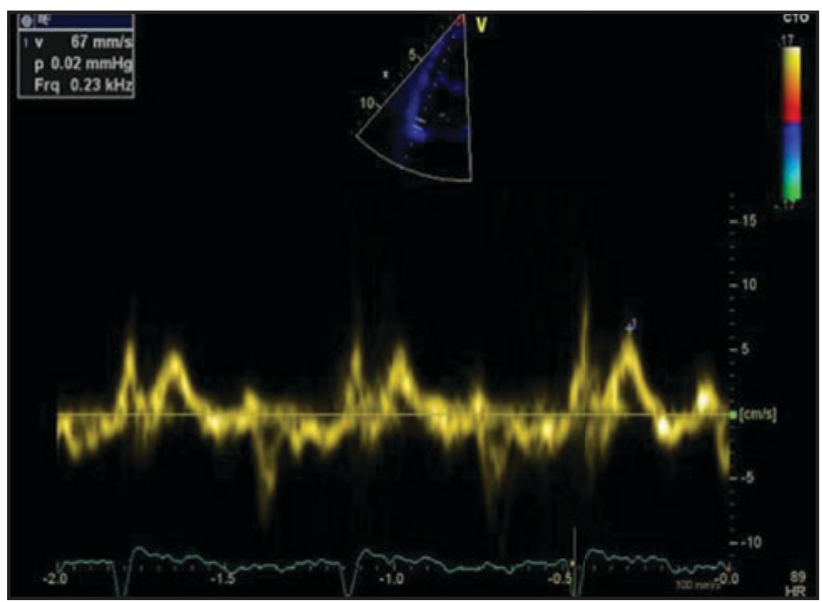

Figure 4. Trans-thoracic echocardiography, 4-chambers apical section, Tissue Doppler examination, with sample placed at the free $R V$ wall level, indicating longitudinal systolic dysfunction of $R V$ - S free wall $R V: 6,7 \mathrm{~cm} / \mathrm{s}$. 


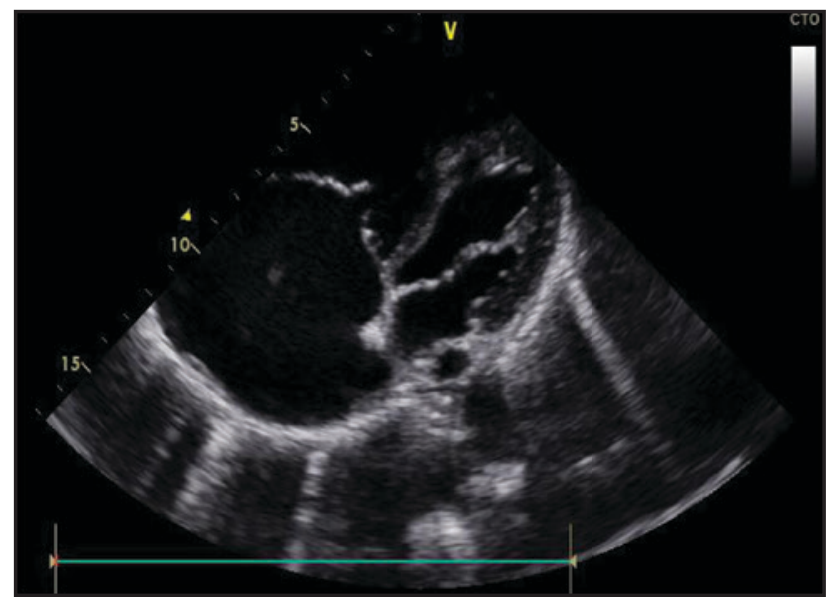

Figure 5. Trans-thoracic echo-cardiography, modified apical section, 2D examination underlining dilated right cavities and $L V$ with reduced dimensions as compared to $R V$.

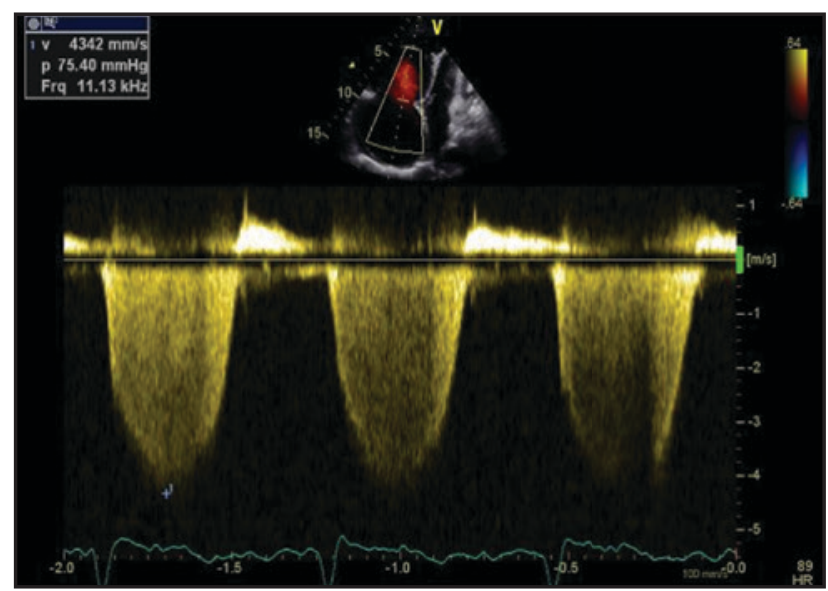

Figure 6. Trans-thoracic echo-cardiography, 4-chambers apical section; Continual Doppler examination at the tricuspid regurgitation jet level underlining the $92 \mathrm{mmHg}$ gradient between the right ventricle and the right atrium, allowing estimation of systolic pressure value within the pulmonary artery of $120 \mathrm{mmHg}$.

Computed thoracic angio-tomography with contrast substance (angio-CT) presents thrombosis at the origin of the right pulmonary artery with extension on the right medium lumbar artery to the periphery, with possible pulmonary infarction within the right inferior lobe and left petrified pleurisy.

Given these data, the following diagnosis was established:

1. Severe chronic thromboembolic pulmonary hypertension, with partially obstructive thrombosis within the right pulmonary artery

2. Congestive cardiac failure, functional class IV

3. Severe functional tricuspidian insufficiency

4. Moderate pulmonary insufficiency

5. Pleurisy and left pachypleuritis

6. Deep venous thrombosis at the level of the right inferior limb in antecedents.

We considered our patient having indication for pulmonary thromboendarterectomy, so we performed coronarography and cardiac right catheterization. The cardiac right catheterization confirms the existence of increased pressures in the pulmonary circulation (pulmonary artery systolic pressure $=110 \mathrm{mmHg}$, medium pulmonary artery pressure $=60 \mathrm{mmHg}$ ) with increased total pulmonary resistance and diminished cardiac index $1,35 \mathrm{l} / \mathrm{min} / \mathrm{m}^{2}$. The coronarography showed normal epicardial coronary arteries and excluded other congenital defects.

\section{Discussions}

Current data [1] suggest that our patient was a potential candidate for the thrombendarterectomy surgery, so we sent him towards a reference tertiary Cardio-Vascular Diseases Centre. Pre-surgical reevaluation and the pneumology investigations effectuated within this center have argued the terms of benefit and prognosis of this patient.

The patient presents negative preoperative prognostic factors with increased risk of hemodynamic instability [2]: severe systolic dysfunction of the right ventricle (ejection fraction of the right ventricle $<20 \%$ ), increased natriuretic atrial peptide (BNP), distal micro-vascular disorder, respiratory severe restriction with severely low gas transfer at the level of the alveolar-capillary membrane. The combination of persistent macro-vascular obstruction (the thrombus in the pulmonary artery) with the development of the micro-vascular arteriopathy explains the clinical aspect of severe pulmonary hypertension. Increased pulmonary vascular resistances, invasively 
determined, are considered an independent mortality risk factor [3], and in the case of our patient the presence of elevated pulmonary vascular resistances $>600$ dynes $/ \mathrm{cm}^{3}$ in association with the macrovascular affection has made him an ineligible candidate for the pulmonary thromboendarterectomy, estimating a higher postoperative risk of mortality and the persistence of the post-thromboendarterectomy pulmonary hypertension. Considering these data, and in particular the micro-vascular disease, we choose the specific combination therapy $[3,4]$ with inhibitors of Phosphodiesterase-5 (Sildenafil 20mg x3/day) and endothelin receptor antagonists (Bosentan oral 62, $5 \mathrm{mg} \times 2 /$ day) along with standard medical treatment with diuretics, oral anticoagulant and hyperbaric medicine. The option for the specific therapy for arterial pulmonary hypertension was argued by the physiopathologic aspects of micro-vascular affectation from the thromboembolic pulmonary hypertension. The main physiopathologic mechanism in the thromboembolic pulmonary hypertension is not represented by the formation of thrombus, but rather from its poor resolution; the pulmonary embolism as a single or repetitive episode is the triggering event followed by a vascular remodeling [5]. In time, there occurs a distal micro-vascular injury similar to that of an idiopathic pulmonary hypertension characterized by plexiform lesions contributing to the pressure load of the right heart chambers and the progression of pulmonary hypertension $[6,7]$.

Therapeutic alternatives available at this moment are represented by the atrial balloon septostomy, as a "bridge" intervention to the cardiopulmonary transplant [8], but considering the favorable evolution of the patient under the specific combination therapy with Phosphodiesterase-5 inhibitors and endothelin receptors antagonists, with the clinic and hemodynamic improvement of pulmonary pressures, the interventional treatment to realize the inter-atrial communication was temporized, the patient being included on the list for cardio-pulmonary transplant.

\section{Case particularity}

A particularity of the presented case is the fact that, on repeating computed thoracic angiotomography with contrast substance after 6 months of medical treatment, patient presents elements of a favorable prognosis, i.e. the development of systemic collaterals and dilation of systemic arteries as response to the pulmonary hypo-perfusion (decreased pulmonary flow, hypoxemia, fibrosis and inflammation). The favorable answer to the specific combined therapy, together with the development of multiple collaterals constitutes the arguments for the favorable clinical evolution of our patient, with an obvious improvement of the right cardiac failure phenomena.

\section{References:}

1. Jenkins, D. (2015). Pulmonary endarterectomy: the potentially curative treatment for patients with chronic thromboembolic pulmonary hypertension. Eur Respir Rev, 24(136), 263-271. doi: 10.1183/16000617.00000815

2. Kim, N.H., Delcroix, M., Jenkins, D.P., Channick, R., Dartevelle, P., Jansa, P., Lang, I., Madani, M.M., Ogino, H., Pengo, V., \& Mayer, E. (2013). Chronic thromboembolic pulmonary hypertension. J Am Coll Cardiol, 62(25 Suppl), D92-99. doi: 10.1016/j.jacc.2013.10.024.

3. Galie, N., Humbert, M., Vachiery, J.L., Gibbs, S., Lang, I., Torbicki, A., Simonneau, G., Peacock, A., Vonk Noordegraaf, A., Beghetti, M., Ghofrani, A., Gomez Sanchez, M.A., Hansmann, G., Klepetko, W., Lancellotti, P., Matucci, M., McDonagh, T., Pierard, L.A., Trindade, P.T., Zompatori, M., \& Hoeper, M. (2015). 2015 ESC/ ERS Guidelines for the diagnosis and treatment of pulmonary hypertension: The Joint Task Force for the Diagnosis and Treatment of Pulmonary 
Hypertension of the European Society of Cardiology (ESC) and the European Respiratory Society (ERS): Endorsed by: Association for European Paediatric and Congenital Cardiology (AEPC), International Society for Heart and Lung Transplantation (ISHLT). Eur Respir J, 46(4), 903-975. doi: 10.1183/13993003.010322015.

4. Galie, N., Palazzini, M., \& Manes, A. (2010). Pulmonary arterial hypertension: from the kingdom of the near-dead to multiple clinical trial meta-analyses. Eur Heart J, 31(17), 20802086. doi: 10.1093/eurheartj/ehq152

5. Hoeper, M.M., Madani, M.M., Nakanishi, N., Meyer, B., Cebotari, S., \& Rubin, L.J. (2014). Chronic thromboembolic pulmonary hypertension. Lancet Respir Med, 2(7), 573-582. doi: 10.1016/S2213-2600(14)70089-X.
6. Simonneau, G., Gatzoulis, M.A., Adatia, I., Celermajer, D., Denton, C., Ghofrani, A., Gomez Sanchez, M.A., Krishna Kumar, R., Landzberg, M., Machado, R.F., Olschewski, H., Robbins, I.M., \& Souza, R. (2013). Updated clinical classification of pulmonary hypertension. $J$ Am Coll Cardiol, 62(25 Suppl), D34-41. doi: 10.1016/j.jacc.2013.10.029.

7. Chin, K.M., Kim, N.H., \& Rubin, L.J. (2005). The right ventricle in pulmonary hypertension. Coron Artery Dis, 16(1), 13-18.

8. Al Maluli, H., DeStephan, C.M., Alvarez, R.J., Jr., \& Sandoval, J. (2015). Atrial Septostomy: A Contemporary Review. Clin Cardiol, 38(6), 395400. doi: $10.1002 /$ clc. 22398 